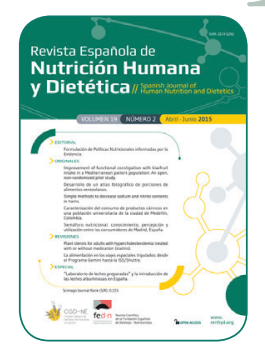

\title{
ORIGINAL
}

\section{Caracterización del consumo de productos cárnicos en una población universitaria de la ciudad de Medellín, Colombia}

\author{
Holmes Rodríguez ${ }^{a, *}$, Luis Fernando Restrepo ${ }^{b}$, Luz Amparo Urango ${ }^{c}$ \\ a Grupo de Investigación GaMMA, Facultad de Ciencias Agrarias, Universidad de Antioquía, Colombia. \\ b Grupo de Investigación GISER, Facultad de Ciencias Agrarias, Universidad de Antioquía, Colombia. \\ C Escuela de Nutrición y Dietética, Universidad de Antioquía, Colombia. \\ *holmes.rodriguez@udea.edu.co
}

Recibido el 16 de enero de 2015; aceptado el 4 de mayo de 2015

PALABRAS CLAVE

Carne;

Población universitaria;

Factores

socioeconómicos;

Conocimientos nutricionales:

Preferencias

alimentarias;

Hábitos de consumo.
Caracterización del consumo de productos cárnicos en una población universitaria de la ciudad de Medellín, Colombia

\section{RESUMEN}

Introducción: El estudio de estilos de vida saludable en universitarios es parte de las estrategias institucionales para reducir la prevalencia de sobrepeso y obesidad. Por tal razón, el objetivo de esta investigación fue caracterizar en la población universitaria de la ciudad de Medellín, Colombia, el consumo y el conocimiento referente a la importancia del consumo adecuado de productos cárnicos.

Material y Métodos: El tamaño de la muestra fue de 400 estudiantes, seleccionados aleatoriamente de acuerdo con los factores de ponderación: género, nivel socioeconómico y tipo de universidad, teniendo en cuenta un nivel de confiabilidad del 95\% y un error máximo permisible del $5 \%$.

Resultados: Se encontró que el pollo es la carne preferida por los universitarios, seguido del pescado; la carne de cordero es la de menor preferencia; se encontró mayor consumo en el género masculino $(p<0,05)$ de pescado, conejo y cabra. Con respecto al gusto que poseen los estudiantes por los diferentes cárnicos, se encontró mayor gusto entre los hombres $(p<0,05)$ $y$ en las universidades públicas $(p<0,0001)$. En cuanto a los conocimientos sobre el valor nutricional, las mujeres tienen un mayor nivel que los hombres $(p<0,05)$ y los estudiantes de estrato bajo son los que menos conocen sobre las propiedades de los cárnicos $(p<0,05)$.

Conclusiones: Teniendo en cuenta el bajo conocimiento de los universitarios sobre la importancia del consumo de cárnicos, es necesario implementar programas de educación e intervención para mejorar sus hábitos alimenticios. 
Characterization of meat product consumption in a college population of Medellin, Colombia

\section{KEYWORDS}

Meat;

College students;

Socioeconomic

status;

Nutrition

knowledge;

Food preferences;

Consumption habits.

\section{ABSTRACT}

Introduction: Study of healthy lifestyles in college students, is part of institutional strategies to reduce prevalence of overweight and obesity. For this reason, the aim of this research was to characterize the consumption and knowledge of university students at Medellin, Colombia, related to consumption of meat products.

Material and Methods: Sample size was 400 students, randomly selected according to the factors: gender, socioeconomic status and type of university (public or private), considering a confidence level of $95 \%$ and a maximum permissible error of $5 \%$.

Results: Chicken meat followed by fish as preferred meat, was found, lamb is the least preferred; higher consumption in males of fish, rabbit and goat was found $(p<0.05)$. With regard to preferences about meat, higher preference by men $(p<0.05)$ and by public universities $(p<0.0001)$ was found. About meat nutritional value's knowledge, higher levels in women than men was found $(p<0.05)$; students from lower socioeconomic status have the least knowledge about the properties of meat $(P<0.05)$.

Conclusions: Considering the low awareness of college students on the importance of eating meat, to implement education programs and intervention to improve dietary habits is necessary.

\section{CITA}

Rodríguez-Espinosa H. Caracterización del consumo de productos cárnicos en una población universitaria de la ciudad de Medellín, Colombia. Rev Esp Nutr Hum Diet. 2015; 19(2): 90 - 96. DOI: 10.14306/renhyd.19.2.147

\section{INTRODUCCIÓN}

Los resultados de la Encuesta Nacional de la Situación Nutricional en Colombia - ENSIN 2010¹ indican que la población colombiana no practica una alimentación saludable. Las cifras muestran deficiencias en la ingesta de los principales grupos de alimentos en la población entre 5 y 64 años; se encontró que un $39 \%$ no consume lácteos diariamente, un $33 \%$ no consume frutas a diario, un $72 \%$ no consume hortalizas y un $14 \%$ no consume carne o huevos diariamente.

Estas prácticas de alimentación no saludable han conllevado a un aumento entre 2005 y 2010 en la prevalencia de sobrepeso y obesidad en jóvenes de 18 a 29 años, alcanzando niveles de $22,8 \%$ y $7,8 \%$ respectivamente, cifras que para adultos mayores a 30 años alcanzan niveles superiores al $40 \%$ en sobrepeso y $18 \%$ en obesidad ${ }^{1}$. A este respecto, la literatura científica reporta el impacto positivo que han tenido campañas gubernamentales con participación comunitaria en hábitos saludables como el aumento del consumo de frutas y verduras, de alimentos bajos en grasa y la reducción del consumo de sal, asociados a la reducción de factores de riesgo de enfermedades cardiovasculares ${ }^{2}$.

Por otra parte, los estudios en población universitaria sobre hábitos alimenticios han encontrado que la forma en que se alimentan los estudiantes tiende a apartarse de hábitos saludables ${ }^{3}$, especialmente por razones asociadas con la falta de tiempo y modificaciones en la alimentación resultantes del estrés o el consumo de alimentos con alta densidad energética ${ }^{4,5}$, decisiones de consumo asociadas al sabor y no tanto a motivos nutricionales ${ }^{6}$, la influencia del contexto social, cultural y educativo ${ }^{7}$, residencia fuera del hogar relacionada con mayor consumo de comidas rápidas ${ }^{8}$, y la poca aplicación de los conocimientos adquiridos ${ }^{9}$; esta situación sumada a cambios en el estilo de vida -como el aumento del sedentarismo-, ha conllevado a un incremento del riesgo de 
padecer enfermedades crónicas en este grupo poblacional ${ }^{5}$.

Adicionalmente, estudios realizados en población universitaria $^{10}$ han encontrado entre los hábitos poco saludables el consumo inadecuado de alimentos cárnicos, lo cual ha conllevado a la ingesta excesiva de grasa saturada, colesterol y proteína animal, asociada a dicho consumo. Por lo anterior, y teniendo en cuenta que las estrategias institucionales para el bienestar apuntan al estudio de estilos de vida saludable en universitarios, incluyendo aspectos como conocimientos y hábitos alimentarios ${ }^{11}$, el presente estudio tuvo como objetivo caracterizar en población universitaria de la ciudad de Medellín, Colombia, el consumo y el conocimiento referente a la importancia del consumo adecuado de productos cárnicos, entendidos éstos como carnes de vacuno, cerdo, ovino, caprino, conejo y carnes frías, incluyendo además el pescado, por estar asociado a la decisión de compra del consumidor bajo un mismo sistema de demanda ${ }^{12}$. Esta caracterización permitirá establecer posibles asociaciones entre el consumo y variables como género, nivel socioeconómico y tipo de universidad.

\section{MATERIAL Y MÉTODOS}

\section{Diseño, población y muestra}

Se realizó un estudio descriptivo multidimensional de tipo transversal, en el cual la población objetivo fueron estudiantes universitarios de la ciudad de Medellín, Colombia. Se utilizó un nivel de confiabilidad del 95\% y un error máximo permisible del $5 \%$, en el cual los parámetros estimados $P$ (proporción de personas en la población objetivo que poseen la característica de interés: consumo de carne) y $Q$ (complemento de $\mathrm{P}$, es decir $\mathrm{Q}=1-\mathrm{P}$ ) adoptaron el valor del $50 \%$, debido a que no se poseen estudios anteriores sobre el consumo de carnes en la población de universitarios en la ciudad de Medellín.

Teniendo en cuenta un marco muestral conformado por 150.000 matriculados $^{13}$, el tamaño mínimo estimado de la muestra fue de 384 individuos pertenecientes a universidades públicas y privadas de la ciudad de Medellín, Colombia, cuya edad osciló entre los 17 y 25 años de edad. Se realizó un muestreo aleatorio de proporciones en forma triplemente estratificada por género, nivel socioeconómico y tipo de universidad.

\section{Variables e instrumentos}

Para llevar a cabo el proceso investigativo, se diseñó una encuesta de tipo estructurado en función de los objetivos planteados -la cual fue ajustada de acuerdo con las recomendaciones de Nutricionistas y Dietistas expertos en el tema- y teniendo en cuenta variables de tipo cualitativo asociado a patrones probabilísticos, multinomial y binomial. Las preguntas relacionadas con el instrumento fueron: le gusta consumir cárnicos, conoce las propiedades alimenticias que ellos poseen, conoce su importancia para la salud, sabe conservar los cárnicos, consumo y frecuencia de consumo de los siguientes cárnicos (carne de res, carne de pollo, pescado, carne de cerdo, carne de conejo, carne de cabra, carnes frías, carne de cordero) teniendo en cuenta la escala frecuente correspondiente a "por lo menos una vez por semana", "por lo menos una vez al mes" y "rara vez menos de una vez al mes"; de igual manera se efectuaron preguntas relacionadas con la forma cómo le gusta la preparación de la carne (asada, cocida, frita, cruda, a la plancha).

\section{Recolección de datos}

La encuesta fue aplicada con la participación voluntaria de los estudiantes, pertenecientes a universidades públicas y privadas de la ciudad de Medellín, Colombia (200 estudiantes de universidad pública, 200 estudiantes de universidad privada, eligiéndose en cada tipo de universidad 100 mujeres y 100 hombres), cuya edad osciló entre los 17 a 25 años. El abordaje de los datos se realizó aplicando la encuesta de manera presencial, con cita previa. La recolección de los datos se realizó en el período mayo - octubre de 2014.

\section{Análisis estadístico}

Para el análisis estadístico de los datos, se empleó el modelo lineal general, incorporando el análisis multivariado de la varianza MANOVA con contraste canónico ortogonal, estableciendo la dimensionalidad de la comparación multidimensional, por medio del criterio de máxima verosimilitud observando el mayor valor propio significativo. Anotando que el MANOVA se realizó con base en la información generada a partir del constructor de los factores cuya naturaleza es continua y utilizando la técnica de contrastes Z; adicionalmente se elaboraron distribuciones de frecuencia de tipo univariado y bivariado mediante tablas de contingencia. El nivel de significancia o error tipo I utilizado en todo el estudio fue del 5\%. El análisis se realizó utilizando el paquete estadístico SAS versión 9.0. 


\section{RESULTADOS}

El pollo es la carne preferida por los universitarios de la ciudad de Medellín (38,6\%), seguido de pescado (37,3\%) y cerdo (17,4\%); la carne de cordero es la de menor preferencia $(6,7 \%)$. Se encontró mayor consumo en el género masculino $(p<0,05)$ de pescado, conejo y cabra (Tabla 1$)$.

El $32 \%$ de los hombres y $13 \%$ de las mujeres manifestaron que su religión les impide consumir ciertos tipos de cárnicos; el $35,5 \%$ de las mujeres y $24 \%$ de los hombres consideran que los cárnicos son cancerígenos; al $13 \%$ de las mujeres y $16 \%$ de los hombres su nivel de ingresos les impide consumir cárnicos de manera frecuente; el $55,5 \%$ de las mujeres y $58,6 \%$ de los hombres consideran que los cárnicos son reemplazables.

A un alto porcentaje de estudiantes les gusta consumir carne; se encontró diferencia altamente significativa $(p<0,0001)$ entre géneros y entre tipos de universidad; siendo mayor el gusto por este alimento en los hombres y en las universidades públicas; no se encontró diferencia estadística entre estratos (Tabla 2). En cuanto al conocimiento de las propiedades alimenticias, se encontró diferencia significativa $(p<0,05)$ entre géneros y entre niveles socioeconómicos, siendo menor el conocimiento en hombre y en el nivel socioeconómico más bajo. Frente al conocimiento en la conservación de los cárnicos, se detectó diferencia significativa $(p<0,05)$ entre géneros, siendo mayor el conocimiento por parte de los hombres.

Tabla 1. Consumo y frecuencia de consumo de cárnicos por género.

$\begin{array}{l:c:c:c:l}\text { Cárnico } & \text { Masculino } & \text { Ingiere } & \text { Femenino } & \text { Ingiere } \\ \text { Carne de res } & 57,2 \mathrm{a} & \text { Frecuente } & 60,6 \mathrm{a} & \text { Frecuente } \\ \text { Carne de pollo } & 98,2 \mathrm{a} & \text { Frecuente } & 94,0 \mathrm{a} & \text { Frecuente } \\ \text { Pescado } & 93,6 \mathrm{a} & \text { Mensual } & 79,1 \mathrm{~b} & \text { Mensual } \\ \text { Carne de cerdo } & 85,2 \mathrm{a} & \text { Frecuente } & 85,3 \mathrm{a} & \text { Frecuente } \\ \text { Carne de conejo } & 77,7 \mathrm{a} & \text { Rara Vez } & 45,0 \mathrm{~b} & \text { Rara Vez } \\ \text { Carne de cabra } & 58,7 \mathrm{a} & \text { Rara Vez } & 39,0 \mathrm{~b} & \text { Rara Vez } \\ \text { Carnes frías } & 94,5 \mathrm{a} & \text { Frecuente } & 90,0 \mathrm{a} & \text { Frecuente } \\ \text { Carne de cordero } & 4,9 \mathrm{a} & \text { Rara Vez } & 6,0 \mathrm{a} & \text { Rara Vez }\end{array}$

a • b: Letras distintas indican diferencia significativa entre géneros. Se empleó el método Z.

Tabla 2. Gustos y conocimientos referentes al consumo de cárnicos (\%).

\begin{tabular}{|c|c|c|c|c|c|c|c|}
\hline & \multicolumn{2}{|c|}{ Género } & \multicolumn{3}{|c|}{ Estrato } & \multicolumn{2}{|c|}{ Tipo de Universidad } \\
\hline & M & $\mathbf{F}$ & Alto & Medio & Bajo & Pública & Privada \\
\hline Le gusta consumir cárnicos & 72,3 a & $66,3 \mathrm{~b}$ & 72,0 a & 67,9 a & 69,3 a & 71,4 a & $63,2 \mathrm{~b}$ \\
\hline Conoce sus propiedades alimenticias & $20,5 \mathrm{~b}$ & 42,0 a & 37,0 a & 32,4 a & 19,6 b & $30,5 \mathrm{~b}$ & 37,6 a \\
\hline Conoce su importancia para la salud & $31,0 \mathrm{~b}$ & 47,1 a & 46,5 a & 42,5 a & 29,6 b & 32,6 b & 44,2 a \\
\hline Sabe conservar los cárnicos & 76,1 a & $67,3 \mathrm{~b}$ & 76,9 a & $68,4 \mathrm{~b}$ & $66,5 \mathrm{~b}$ & 69,2 b & 75,6 a \\
\hline Valor de $p$ derivado del MANOVA & \multicolumn{2}{|c|}{0,0001} & \multicolumn{3}{|c|}{0,5826} & \multicolumn{2}{|c|}{0,0001} \\
\hline
\end{tabular}

a • b: Letras distintas indican diferencia significativa entre géneros, entre estratos y entre tipos de universidad. Se empleó el modelo lineal general (GLM) para el MANOVA, teniendo en cuenta el constructor de los factores. 
Con relación al conocimiento sobre la importancia de los cárnicos para la salud, se detectó diferencia significativa $(p<0,05)$ entre géneros, a favor del sexo femenino.

En cuanto a la preparación de la carne, se encontró que hombres y mujeres prefieren la carne asada al carbón y en segundo lugar a la plancha; la carne cruda es la de menor preferencia para hombres y mujeres (Tabla 3). Se encontró diferencia significativa ente géneros en cuanto a la preferencia por carne asada, a la plancha y cocida $(p<0,05)$.

\section{DISCUSIÓN}

Para el contexto de la población universitaria de la ciudad de Medellín, se encontró que el pollo es la carne preferida por los universitarios, seguido del pescado; la carne de cordero es la de menor preferencia. Estos resultados concuerdan con otros hallazgos indicando que los universitarios cuidan su salud mediante la alimentación en mayor proporción que mediante el ejercicio y la buena alimentación implica el consumo de mayor cantidad de carnes blancas que rojas ${ }^{14}$. No se encontró diferencia significativa entre géneros en los hábitos de consumo, resultado que contrasta con otros autores que encontraron que las mujeres tienen hábitos de consumo más saludables que los hombres 6,15 .

La diferencia encontrada entre tipos de universidades en el gusto por el consumo de carne, con un mayor porcentaje de estudiantes de universidades públicas a quienes les consumir carne puede estar asociada con la tendencia actual hacia una alimentación más saludable que implica menor consumo de carnes rojas y mayor consumo de vegetales, la cual puede estar más arraigada en las universidades privadas en las cuales los estudiantes tienen mayores niveles económicos.

Se destaca la alta preferencia de consumo de pescado, que contrasta con otros resultados encontrados en población universitaria con una baja proporción de ingesta proteica procedente del grupo de pescados ${ }^{10}$. Un factor que puede incidir en el alto consumo de pescado, es el consumo regular de alimentos en las cafeterías universitarias, por cuanto la literatura reporta que se ha encontrado asociación entre éste y el incremento en el consumo de carne y pescado, independiente del nivel socioeconómico o creencias sobre dieta y salud ${ }^{16}$.

La frecuencia de consumo de carne de cerdo concuerda con los hallazgos de otros autores que han encontrado una gran preferencia por este tipo de carne en la población estudiada, la cual puede ser incorporada en la dieta sin efectos adversos en la salud, concluyendo que puede ser disfrutada a diario en la dieta, si se educa a la población ${ }^{17}$.

Estos resultados también muestran un alto gusto por la carne y alta frecuencia de consumo, al igual que un bajo porcentaje de estudiantes que indican tener limitaciones en el consumo frecuente de carne debido a su nivel de ingreso. Al respecto, otros estudios reportan que la alta frecuencia de consumo de carne en jóvenes está asociada con mayores niveles de satisfacción con la alimentación y de satisfacción con la vida ${ }^{18}$. A diferencia de estos resultados, otras investigaciones encontraron que cerca del 30\% de estudiantes universitarios, tenían bajo o muy bajo consumo de proteína en su dieta ${ }^{19}$.

En cuanto al mayor gusto por la carne por parte de los hombres, estos resultados coinciden con los hallazgos de otros autores, quienes encontraron que los hombres regularmente consumen más carne que las mujeres ${ }^{20}$ y que los hombres universitarios presentan mayor sobre adecuación en el consumo de proteínas, al compararlos con las mujeres.

Tabla 3. Consumo y frecuencia de consumo de cárnicos por género.

\begin{tabular}{l|c:c} 
Preferencia & Masculino \% & Femenino \% \\
Cocida & $5,0 \mathrm{a}$ & $2,1 \mathrm{~b}$ \\
Frita & $13,8 \mathrm{a}$ & $11,2 \mathrm{a}$ \\
Asada & $42,4 \mathrm{~b}$ & $55,5 \mathrm{a}$ \\
A la plancha & $36,8 \mathrm{a}$ & $30,0 \mathrm{~b}$ \\
Cruda & $2,0 \mathrm{a}$ & $1,2 \mathrm{a}$
\end{tabular}


Estos resultados, sumados a otros hallazgos sobre alto consumo de frutas en población universitaria, podrían conducir a apreciaciones relacionadas con una dieta de los universitarios de Medellín con características de una alimentación saludable ${ }^{21}$; sin embargo, este estudio pone en evidencia un factor de riesgo como la alta frecuencia de consumo de carnes frías (94,5\% hombres, 90,0\% mujeres) que amerita estudios complementarios, por cuanto otros estudios reportan que la mayoría de personas que cuidan su salud consumen menos embutidos frente a aquellos que no tienen este hábito, debido a que los embutidos son percibidos como malos para la salud ${ }^{14}$. En el mismo sentido, otros autores encontraron que las personas con mayor intención de consumir comida saludable adquirían comida no saludable como los embutidos, con menor frecuencia22.

En relación con los conocimientos sobre el valor nutricional, se encontró diferencia entre niveles socioeconómicos; los estudiantes de estrato bajo son los que menos conocen sobre las propiedades de los cárnicos. El conocimiento de las propiedades alimenticias de los cárnicos y su importancia en la salud es bajo; se detectó diferencia a favor del sexo femenino; por el contrario se encontró diferencia significativa a favor de los hombres en cuanto al mayor conocimiento sobre la forma de conservar los cárnicos. Estos resultados concuerdan con los hallazgos de otros autores que encontraron una diferencia significativa a favor de las mujeres en cuanto a la importancia que otorgan al contenido nutricional de los alimentos ${ }^{23}$. Otros estudios encontraron que los universitarios manifiestan estar poco informados en asuntos relacionados con alimentación y nutrición?.

Frente a los hábitos alimenticios de los universitarios, otros estudios encontraron correlación entre éstos y la prevalencia de fatiga, lo cual está relacionado también con el rendimiento académico ${ }^{24}$. Igualmente se ha encontrado asociación entre el estrés y los patrones de consumo de universitarios, con una reducción en el consumo de comidas regulares, que incluyen carne y comidas balanceadas, en hombres y mujeres, a favor del consumo de comidas rápidas en períodos de estrés ${ }^{25}$; siendo estos períodos mayores en estudiantes de primer año, lo cual está asociado con la selección de alimentos poco saludables, especialmente en los hombres ${ }^{26}$.

Por lo anterior se plantea la necesidad de implementar programas orientados a enseñar buenos hábitos alimenticios a los estudiantes y sus familias; entendiendo que en los hábitos alimentarios de estudiantes universitarios no predomina un patrón racional de análisis nutricional, sino un patrón relacionado con aspectos sociales a los cuales se asocia el consumo de alimentos ${ }^{18,27}$. Adicionalmente, como son muy pocos los cambios en los hábitos típicos de consumo de los estudiantes a lo largo de su estancia en la universidad ${ }^{28}$, programas de educación con información sobre el aporte nutricional de las comidas para mejorar su comportamiento en cuanto a la alimentación saludable ${ }^{29}$ desde el inicio de la carrera, pueden mejorar la calidad de la dieta de los universitarios y por consiguiente su desempeño académico.

Estos programas educativos de promoción de hábitos alimenticios saludables, deben ser complementados con acompañamiento al estudiante para que se produzca la modificación real de los hábitos alimenticios poco saludables y lograr una dieta equilibrada ${ }^{7}$. Adicionalmente, como la satisfacción con la alimentación está más asociada con el disfrute, que con motivos nutricionales o de salud, se debe tener en cuenta este aspecto en el diseño de las estrategias de promoción del consumo saludable ${ }^{18}$.

Finalmente, este estudio representa una primera aproximación al entendimiento de las características del consumo de cárnicos en población universitaria que, por su diseño, ha permitido obtener inferencias estadísticas, aunque tiene por limitación que no se calculó el consumo estimado de cárnicos. Por lo anterior, se sugiere adelantar nuevas investigaciones para profundizar en el conocimiento de los hábitos de consumo de los universitarios y en especial del consumo de carnes frías, por cuanto es elevado y representa un factor de riesgo en un esquema de alimentación saludable.

\section{Conclusiones}

Los resultados del presente estudio indican que a los universitarios les gusta consumir carne; con un mayor gusto por parte de los hombres y sin diferencia entre niveles socioeconómicos o tipos de universidad. El pollo es la carne preferida por los universitarios -tanto hombres como mujeres-, seguido del pescado en el cual se encontró un mayor consumo por parte de los hombres. En cuanto a los conocimientos sobre el valor nutricional, las mujeres tienen un mayor conocimiento que los hombres y los estudiantes de estrato bajo son los que menos conocen sobre las propiedades de los cárnicos. Las deficiencias en el conocimiento sobre la importancia del consumo de cárnicos permiten inferir la necesidad de implementar programas de educación e intervención para mejorar los hábitos alimenticios de los universitarios, teniendo en cuenta su relación con la fatiga y el estrés y de estos factores con el rendimiento académico. 


\section{BIBLIOGRAFÍA}

1. Instituto Colombiano de Bienestar Familiar, Ministerio de la Protección Social, Instituto Nacional de Salud \& Profamilia. Encuesta Nacional de la Situación Nutricional en Colombia 2010. 1-512; 2011.

2. Roos G, Lean M, Anderson A. Dietary interventions in Finland, Norway and Sweden: nutrition policies and strategies. J Hum Nutr Diet. 2002; 15(2): 99-110.

3. Ortiz-Moncada R, Norte Navarro Al, Zaragoza Marti A, Fernández Sáez J, Davó Blanes MC. ¿Siguen patrones de dieta mediterránea los universitarios españoles? Nutr Hosp. 2012; 27(6): 1952-9.

4. Troncoso C, Amaya JP. Factores sociales en las conductas alimentarias de estudiantes universitarios. Rev Chil Nutr. 2009; 36(4): 1090-7

5. Becerra-Bulla F, Pinzón-Villate G, Várgas-Zárate M. Estado nutricional y consumo de alimentos de estudiantes universitarios admitidos a la carrera de medicina Bogotá 2010-2011. Rev Fac Med. 2012; 60: 3-12.

6. Schnettler B, Miranda H, Sepúlveda J, Denegri M. Satisfacción con la alimentación y la vida, un estudio exploratorio en estudiantes de la universidad de la frontera, Temuco-Chile. Psicol e Soc. 2011; 23(2), 426-35.

7. Montero Bravo A, Ubeda Martín N, García González A. Evaluation of dietary habits of a population of university students in relation with their nutritional knowledge. Nutr Hosp. 2006; 21(4): 466-73.

8. Schnettler B, Denegri M, Miranda H, Sepúlveda J, Orellana L, Paiva $G$, et al. Hábitos alimentarios y bienestar subjetivo en estudiantes universitarios del sur de Chile. Nutr Hosp. 2013; 28(6): 2221-8.

9. Rizo-Baeza MM, González-Brauer NG, Cortés E. Calidad de la dieta y estilos de vida en estudiantes de Ciencias de la Salud. Nutr Hosp. 2014; 29(1): 153-7.

10. Cervera Burriel F, Serrano Urrea R, Vico García C, Milla Tobarra M, García Meseguer MJ. Hábitos alimentarios y evaluación nutricional en una población universitaria. Nutr Hosp. 2013; 28(2): 438-46

11. Arrivillaga M, Salazar IC, Correa D. Creencias sobre la salud y su relación con las prácticas de riesgo o de protección en jóvenes universitarios. Colomb Med. 2003; 34(4), 186-95.

12. Albisu LM, Gracia A. La demanda de productos cárnicos y pescados en España, aplicación de un sistema de demanda casi ideal (AIDS). Investig Agrar. 1995; 2: 233-52.

13. Ministerio de Educación Nacional. Sistema Nacional de Información de la Educación Superior. Disponible en: http:// www.mineducacion.gov.co/sistemasdeinformacion/1735/w3article-212400.html>
14. Li KK, Concepcion RY, Lee H, Cardinal BJ, Ebbeck V, Woekel $E$, Readdy RT. An examination of sex differences in relation to the eating habits and nutrient intakes of university students. J Nutr Educ Behav. 2012; 44(3): 246-50.

15. Guagliardo V, Lions C, Darmon N, Verger P. Eating at the university canteen. Associations with socioeconomic status and healthier self-reported eating habits in France. Appetite. 2011; 56(1): 90-5.

16. Murphy KJ, Parker B, Dyer KA, Davis CR, Coates AM, Buckley $J D$, et al. A comparison of regular consumption of fresh lean pork, beef and chicken on body composition: a randomized cross-over trial. Nutrients. 2014; 6(2): 682-96.

17. Schnettler B, Miranda $H$, Sepúlveda J, Denegri M, Mora M, Lobos G. Satisfacción con la alimentación en personas Mapuche de la región de la Araucania, Chile. Rev Chil Nutr. 2012; 39(1): 18-29.

18. Vargas-Zárate M, Becerra-Bulla F, Prieto-Suárez E. Evaluación de la ingesta dietética en estudiantes universitarios. Bogotá, Colombia. Rev Salud Pública. 2010; 12(1): 116-25.

19. El Ansari W, Stock C, Mikolajczyk RT. Relationships between food consumption and living arrangements among university students in four European countries - a cross-sectional study. Nutr J. 2012; 11: 28

20. Araya-Quesada $Y$, Jiménez-Robles A, Ivankovich-Guillén C, García-Barquero M. Hábitos de consumo de embutidos en el cantón de San Carlos y el área metropolitana de Costa Rica. Tecnol en Marcha. 2014; 27(4): 113-24.

21. Restrepo LF, Urango LA, Deossa GC. Conocimiento y factores asociados al consumo de frutas por estudiantes universitarios de la ciudad de Medellín, Colombia. Rev Chil Nutr. 2014; 41(3): 236-42

22. Mead E, Gittelsohn J, Roache C, Sharma S. Healthy food intentions and higher socioeconomic status are associated with healthier food choices in an Inuit population. J Hum Nutr Diet. 2010; 23(Suppl 1): 83-91.

23. Morse KL, Driskell JA. Observed sex differences in fast-food consumption and nutrition self-assessments and beliefs of college students. Nutr Res. 2009; 29(3): 173-9.

24. Tanaka M, Mizuno K, Fukuda S, Shigihara Y, Watanabe Y. Relationships between dietary habits and the prevalence of fatigue in medical students. Nutrition. 2008; 24(10): 985-9.

25. Liu C, Xie B, Chou CP, Koprowski C, Zhou D, Palmer P, et al. Perceived stress, depression and food consumption frequency in the college students of China Seven Cities. Physiol Behav. 2007; 92(4): 748-54.

26. Papier K, Ahmed F, Lee P, Wiseman J. Stress and dietary behaviour among first-year university students in Australia: sex differences. Nutrition. 2015; 31(2): 324-30.

27. Cousineau TM, Franko DL, Ciccazzo M, Goldstein M, Rosenthal E. Web-based nutrition education for college students: Is it feasible? Eval Program Plann. 2006; 29(1): 23-33.

28. Driskell JA, Kim YN, Goebel KJ. Few differences found in the typical eating and physical activity habits of lower-level and upper-level university students. J Am Diet Assoc. 2005; 105(5): 798-801.

29. Cooke R, Papadaki A. Nutrition label use mediates the positive relationship between nutrition knowledge and attitudes towards healthy eating with dietary quality among university students in the UK. Appetite. 2014; 83: 297-303. 